\title{
Survey on Minimizing Energy Consumption in Mobile Cloud Computing
}

\author{
C. Arun \\ Ph.D. Research Scholar \\ $P G$ and Research \\ Department of Computer Science \\ Dr. N.G.P. Arts and Science College \\ Coimbatore, Tamil Nadu.
}

\author{
V. Jaiganesh, $\mathrm{PhD}$ \\ Professor \\ $P G$ and Research \\ Department of Computer Science \\ Dr. N.G.P. Arts and Science College \\ Coimbatore, Tamil Nadu.
}

\begin{abstract}
Mobile cloud computing is the thrust research area in the field of recent communication paradigm. Eminent energy conservation strategies are proposed by various researchers in the field of mobile cloud computing. Optimizing energy consumption also plays an important role in mobile cloud computing. This paper reviews the existing research contributions on minimizing energy consumption in mobile cloud computing. It is inferred that maximum of $82 \%$ of energy can be conserved by making use of effective task scheduling method.
\end{abstract}

\section{Keywords}

Mobile cloud computing, energy consumption, task scheduling, optimizing energy, communication

\section{INTRODUCTION}

With the advancement of wireless communication and the boom of technology, mobile devices are widely involved in day-to-day applications. It conceives drastic comfort to the users. Mobile devices now evolved as smart phones that have the ability to fulfill complicated tasks by means of the support of advanced hardware technologies, such as faster CPU, larger memory storage, enhanced LCD / LED screens and higher communication bandwidth such as $4 \mathrm{G}, 5 \mathrm{G}$ and much more. Mobile / smart phone / handheld devices that exist are not capable enough to shore up plentiful computation and communication due to the available battery power. The shorter battery lifetime will certainly hampers the development of cloud based applications.

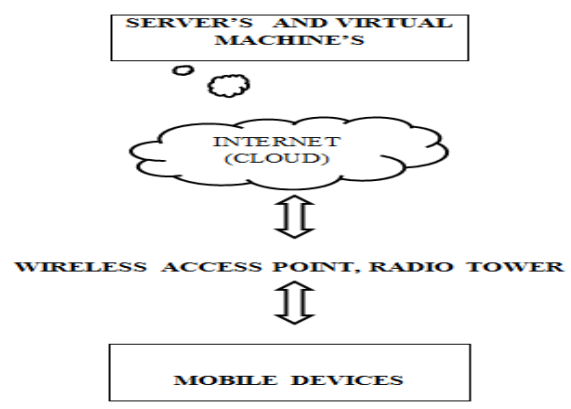

\section{Figure 1: Mobile Computing Architecture}

Accurate modeling of the delay and energy overhead of dynamic voltage and frequency scaling in modern microprocessors [10] is also gives motivation to the energy conservation problem.
Consecutively to get hold of stable and effective network service, mobile devices that are connected with the cloud platform struggles with the problem of energy optimization. Hence design and development of energy efficient mobile cloud computing emerges as the thrust research area in mobile computing. This paper reviews various research works conducted with the aim of providing energy efficient solutions to mobile cloud computing. Fig .1 describe the simple structure of the mobile cloud computing. The mobile devices are communicated with server and virtual machines through internet using wireless access points.

\section{RELATED WORKS}

Tasks offloaded to the cloud for speeding up executions and saving energy results in some additional cost during the communication between cloud and mobile devices. Tundong Liu et. al., proposed an energy-efficient scheduling of tasks[1], where the mobile device offloads appropriate tasks to the cloud via a Wi-Fi access point. An optimal scheduling has been designed for the hybrid task model including sequential tasks and parallel tasks. The proposed scheduling aims to minimize the energy utilization of a mobile device under the constraint of the total completion time for one application in Wi-Fi networks. This task scheduling problem has been reconstructed into a constrained shortest path problem and the LARAC method has been applied to get the approximate optimal solution. The simulation results have shown that the proposed strategy gains similar or better performance compared with Zhang's strategy. The proposed strategy with cloud assistance compared with the local strategy, decreases $81.93 \%$ of energy consumption and $25.70 \%$ of time at most.

Mobile devices join and leaves cloud services frequently, which cause energy inefficiency in a cloud data center. TienDung Nguyen et. al., proposed two approaches to energy efficiency[2]: an Instant Energy Policy (IEP) which finds quickly an appropriate low-power state based on a predicted disconnection time and a Prediction-based Energy Policy (PrEP) to decide when to transit VMs to a low-power state and when to return them to the active state based on each users activity history. The proposed energy policies has been evaluated with simulations using the Social Evolution dataset collected by the MIT Human Dynamics Lab. Power consumption and transition time based on multiple VMs has been measured. It has been observed that the power consumption is $41 \mathrm{w}$ while the CPU usage is $0.25 \%$, and $73 \mathrm{w}$ while the CPU usage increases to $72 \%$. The effects of the number of VMs on transition time have also been studied by increasing the number of running VMs from 1 to 10 . The transition time from the active state to the suspended state and vice versa is measured as 4.5 seconds to 7.5 seconds. The 
transition time from running state to paused state and vice versa adjusts from $1.5 \mathrm{~s}$ to $4.5 \mathrm{~s}$. The transition time from active to the paused state and vice versa adjusts from $1.5 \mathrm{~s}$ to $4.5 \mathrm{~s}$. The energy savings and service delay between IEP and PrEP has also been compared. It has been observed that the average energy consumption of PrEP is lower than both WoEP and IEP, and the average service delay of PrEP which is approximately 3 seconds is also lower than that of IEP which is approximately 5 seconds. The total energy consumption for the methods $\operatorname{PrEP}(\mathrm{j})$, Wo-EP, $\operatorname{PrEP}(\mathrm{j}, \mathrm{s})$ and EP-WoS ranges from a minimum of 54.6 to a maximum of 62.9 , the delay ranges from a minimum of 0 seconds to a maximum of 5.57 seconds, and the power consumption ranges from a minimum of $51.44 \mathrm{w}$ to a maximum of $68.01 \mathrm{w}$. The experimental evaluation concludes that the proposed PrEP approach is providing approximately $20 \%$ power saving and guaranteeing minimal service delay.

One of the challenges in mobile cloud computing is optimizing the tradeoff between system throughput and energy consumption. Being inspired by the feasibility of transmission scheduling for prefetching-friendly or delaytolerant applications, Weiwei Fang et. al., mathematically formulated this problem as a stochastic optimization problem. An online control algorithm MOTET[3], for Mobile device Optimization on the Throughput-Energy Tradeoff using the Lyapunov optimization framework, has been proposed to balance such an energy-performance tradeoff. The proposed algorithm, independently and simultaneously, is able to make control decisions on admission and transmission to maximize a joint utility of the average application energy cost and throughput, without requiring statistical information of traffic arrivals and link bandwidth. A mobile device with three stateof-the-art wireless interfaces; Wi-Fi, 3G-HSDPA, and 4GLTE, has been considered for the performance evaluation of the proposed MOTET algorithm. The link bandwidth traces are the UMICH measurement datasets from 4GTest Project. The MOTET algorithm has been compared with an online algorithm FASTEST. The evaluation shows the power parameters $(\alpha \& \beta)$ for the considered wireless interfaces; Wi$\mathrm{Fi}, 3 \mathrm{G}-\mathrm{HSPDA}$, and 4G-LTE ranges from 51.97 to 137.01 and from 132.86 to 1288.04 respectively, and thus MOTET achieved desired performance tradeoff among application throughput, energy cost and service delay.

Due to the restrictions of wireless bandwidth and device capacity, extra energy waste and latency delay has become a vital consideration while deploying Mobile Cloud Computing. Keke Gai et. al., proposed a dynamic energy-aware cloudlet based mobile cloud computing model (DECM)[4] to solve the additional energy consumptions during the wireless communications by leveraging dynamic cloudlets (DCL)based model. To assign, manage, and optimize the cloudbased infrastructure usages and services DECM uses cloudlets technique to achieve green computing. The proposed algorithm has been evaluated based on a simulation which is a mathematical deduction by comparing proposed DECM approach with traditional cloud computing method. A simulator named DECM-Sim has been used to examine the workload. The proposed algorithm has been evaluated with parameters such as timing units, wireless communication performances, energy consumptions, mobility routes, and switching nodes among different routes. Smart phones with Android 4.4.2 and up operating system have been used for evaluation. Two experiments were made, one to evaluate whether an optimal solution exists at each timing constraint when two-route is deployed and the second to test the operability of DECM in complicated CDLs-connected environment. It has been stated that the experimental results shows that the proposed DECM was efficient in saving energy as well as higher-level wireless service quality.

Chathura M. Sarathchandra Magurawalage et. Al., proposed an architecture [5] for Mobile Cloud Computing (MCC) which includes a cloudlet middle layer between mobile devices and their cloud infrastructure or clones. A data caching mechanism has been introduced at cloudlets for the overall improvement of MCC performance. An offloading algorithm decides whether to offload to a clone or a cloudlet. Simulation results of the proposed work demonstrate the effectiveness and efficiency of the proposed system architecture and the offloading algorithm in terms of response time and energy consumption by a mobile device. CloudSim 2.0 has been used to implement the proposed algorithm. Comparisons have been made among the following three scenarios: mobile only, clone only, and Cloudlet + Clone.

Utilizing MCC, in enabling mobile devices to run complex real-time applications, while keeping high energy efficiency, remains a challenge. Ting Shi et. al., introduced the local mobile clouds formed by nearby mobile devices and a mathematical models of the mobile devices and their applications has been provided [6]. Also a scheduling problem in local mobile clouds has been formulated. The performance of various scheduling algorithms in local mobile clouds has been evaluated by conducting extensive simulations on a local mobile cloud simulation module developed on OMNET++. The metrics, Task completion rate, Average task waiting time, Average energy per successful task has been evaluated in the experiments. The adaptive probabilistic scheduler proposed has the highest completion rate $16.6 \%$ and $3.6 \%$ than the greedy scheduler and the probabilistic scheduler, respectively. It has been stated that the proposed adaptive probabilistic scheduler contain a better chance to offload to a more powerful node with even lower average waiting time, which is 2-4 times shorter than the greedy scheduler. The average energy per successful task of the adaptive probabilistic scheduler is the best.

The method proposed [7] by Mati B. Terefe et. al., differentiates the data intensive and computation intensive components of an application and it performed a multisite offloading in a data and process-centric manner. A novel model has been presented which describes the energy utilization of a multisite application execution and used a discrete time Markov chain (DTMC) in modeling fading wireless mobile channels. A Markov decision process (MDP) framework has been adopted to develop the multisite partitioning problem as a delay constrained, least-cost shortest path problem on a state transition graph. The proposed EMOP (Energy-efficient Multisite Offloading Policy) algorithm that has been built on a Value Iteration Algorithm (VIA), founds the efficient solution to the multisite partitioning problem. The numerical simulation results reveal that the proposed algorithm considered the different potentials of sites in distributing the suitable components to achieve a lower energy cost for data transfer to the cloud from the mobile. A multisite offloading execution using the proposed EMOP algorithm attained a greater reduction on the energy utilization of mobiles when compared to a single site offloading execution. The performance of the proposed EMOP algorithm has been evaluated in terms of energy saving by comparing the results to a single site offloading execution. The energy consumption of an application has been observed with nodes ranging from 10-150, in single site execution and multi site execution. From the simulation results, the proposed EMOP algorithm has 
been found to be an efficient multisite computation offloading approach for mobile devices. It has also been found outperforming the mobile and single site execution with respect to both energy consumption and execution time.

Radhika Loombaa et. al., [8]addressed the trade-off between the quality of the sensed data received by applications and the required energy to transfer data from the mobile handsets, by considering a scheme in which a collaborative sensing middleware intervene between multiple applications requiring sensed data and the mobile handsets located within a particular physical area. An algorithm, Info-Aggregation, has been presented which seeks to maximize the degree to which sensed data transferred from a mobile device can be served to more than one application. The performance has been evaluated in terms of energy consumption. A simulation study was made to assess the performance of the No-Aggregation and Info-Aggregation algorithms, and as a result, the InfoAggregation algorithm effectively reduced the number of active mobile devices and also the volume available for offloading from the sensing environment. The number of applications varied between 50 and 200, varied the number of sensor types requested by applications between 10, 15, 20 and 25 , and varied the number of mobile devices present in the environment in the range of 300-1000 in the experiments. Each experiment has been run 30 times using different random number generator seeds. For each test-case the performance of the Info-Aggregation algorithm has been compared with the No-Aggregation algorithm in terms of the gain in mean battery values of all mobile devices, the difference in volume of data available for offloading and the difference in the mean number of active mobile devices in the sensing area during the planning prospect. The simulation results showed that the number of active devices can be reduced up to more than $50 \%$ when sensed data from mobile devices is shared between multiple interested applications. The mean increase, due to the decreased number of activated mobile devices needed by the Info-Aggregation algorithm to provide the required sensed data to the applications and to the impact of fewer message transmissions due to the use of aggregation, in cumulative residual energy stored in mobile device batteries at the end of the simulated time interval for the Info-Aggregation algorithm has been compared with the No-Aggregation algorithm. As the number of applications increases this effect is improved, as the same sensor data is requested by multiple applications. The evaluation showed that the proposed scheduling algorithm, Info-Aggregation, succeeded in delivering a significant improvement in terms of energy utilization in comparison to a technique that does not employ aggregation. It has also been found that the using truncated Levy Walk mobility model mean improves the performance even when devices move around within the physical area to be sensed.

Partially processed apps in the cloud to minimize the overall energy consumption of smart phones will not necessarily save energy if there is no methodical mechanism to evaluate the effect of offloading an app onto the cloud. A mathematical model has been presented [9] by Salwa Adriana Saab et. al., which represents this energy consumption optimization problem. They proposed dynamic minimum-cut algorithm to dynamically solve the problem while taking security measures into account. They also proposed the free sequence protocol (FSP) which allows for the dynamic execution of apps according to their call graph. The experimental setup is configured with an Amazon EC2 Windows cloud instance with the specifications of $1.7 \mathrm{~GB}$ memory, 1 virtual core with $1 \mathrm{EC} 2$ compute unit, $160 \mathrm{~GB}$ instance storage, 64-bit platform, and moderate I/O performance, a Java server in the cloud, and the modified application has been installed on HTC Nexus One Android smart phone. The experiment studied the effect of the factors: Workload size, Network type, Computation cost, Security measures such as encryption/decryption and offloading constraints, Signal strength, and Call graph structure. The results reveal that the proposed approach saves battery lifetime and enhances performance. The results also depicts the effects of computation cost, network type, security operations, signal strength, workload amount and call graph structure on the optimized overall energy consumption.

K.Huang et. al.,[11] proposed a set of, schemes for controlling CPU cycles for the model of local computing, time division between MPT and offloading for the other model of offloading and model selection. The CSI and CPU cycle information is used to maximizing the probability of successfully computation given data called computing probability under deadline constrains and minimization of energy consumption. The simulation result shows that the proposed method maximizing the energy saving for offloading.

An offloading decision method is investigated by Liu et al [12] to minimize the energy consumption of mobile device with a reasonable time delay and communication quality. An iterative decoupling algorithm that combines Lagrange duality, variable relation, and KKT techniques is proposed for making near-optimal offloading decisions, thereby reducing the computation complexity. The results demonstrated that, with a mobile cloud offloading service, considerable energy consumption for task execution can be saved and the time delay has been reduced.

\section{FINDINGS AND CONCLUSIONS}

Task scheduling, bandwidth allocation, cloud infrastructure, multisite partitioning and middleware schemes are reviewed in this paper that aims in providing energy efficient solutions to the mobile cloud computing. These research works are mainly performed in various mobile cloud computing scenarios which include simulation and dataset. It is inferred that while providing effective task scheduling based mechanism the energy conservation is reduced up to $82 \%$. There exists lot of research scope particularly in the areas of energy efficiency, reducing computation time and cost. Evolutionary algorithms, fuzzy logic, neural networks are some of the thrust research areas that can help in reducing energy consumption. Also several machine learning algorithms may be deployed for reducing the computational time and cost. Further research may be extended by incorporating integrated solution for mobile cloud computing.

\section{REFERENCES}

[1] T. Liu, F. Chen, Y. Ma, and Y. Xie, "An energy-efficient task scheduling for mobile devices based on cloud assistant," Futur. Gener. Comput. Syst., vol. 61, pp. 1$12,2016$.

[2] T. D. Nguyen, P. P. Hung, T. H. Dai, N. H. Quoc, C. T. Huynh, and E. N. Huh, "Prediction-based energy policy for mobile virtual desktop infrastructure in a cloud environment," Inf. Sci. (Ny)., vol. 319, pp. 132-151, 2015.

[3] W. Fang, Y. Li, H. Zhang, N. Xiong, J. Lai, and A. V. Vasilakos, "On the throughput-energy tradeoff for data transmission between cloud and mobile devices," Inf. Sci. (Ny)., vol. 283, pp. 79-93, 2014. 
[4] K. Gai, M. Qiu, H. Zhao, L. Tao, and Z. Zong, "Dynamic energy-aware cloudlet-based mobile cloud computing model for green computing," J. Netw. Comput. Appl., vol. 59, pp. 46-54, 2016.

[5] C. M. Sarathchandra Magurawalage, K. Yang, L. Hu, and J. Zhang, "Energy-efficient and network-aware offloading algorithm for mobile cloud computing," Comput. Networks, vol. 74, no. PB, pp. 22-33, 2014.

[6] T. Shi, M. Yang, X. Li, Q. Lei, and Y. Jiang, "An energy-efficient scheduling scheme for time-constrained tasks in local mobile clouds," Pervasive Mob. Comput., vol. 27, pp. 90-105, 2016.

[7] M. B. Terefe, H. Lee, N. Heo, G. C. Fox, and S. Oh, "Energy-efficient multisite offloading policy using Markov decision process for mobile cloud computing," Pervasive Mob. Comput., vol. 27, pp. 75-89, 2015.

[8] R. Loomba, L. Shi, B. Jennings, R. Friedman, J. Kennedy, and J. Butler, "Energy-aware collaborative sensing for multiple applications in mobile cloud computing," Sustain. Comput. Informatics Syst., vol. 8, pp. 47-59, 2015

[9] S. A. Saab, F. Saab, A. Kayssi, A. Chehab, and I. H. Elhaji, "Partial mobile application offloading to the cloud for energy-efficiency with security measures," Sustain. Comput. Informatics Syst., vol. 8, pp. 38-46, 2015.

[10] S. Park, J. Park, D. shin, “Accurate modeling of the delay and energy overhead of dynamic voltage and frequency scaling in modern microprocessors," IEEE Trans. Comput.-Aided Des. Integr. Circuits Syst. vol. 32, no. 5, pp. 695-708, 2013

[11] K.Huang, C.You, H.Chae, "Energy efficient mobile cloud computing powered by wireless energy transfer", IEEE comm. vol.34, no.5,pp. 1757-1771, May 2016.

[12] K. Liu, J. Peng, H. Li, X. Zhang, W. Liu, "Multi-device task offloading with time-constraints for energy efficiency in mobile cloud computing," Future Generation Computer Systems, vol. 64, pp. 1-14, 2016. 